\title{
Dark energy, chaotic fields, and fundamental constants
}

\author{
Christian Beck \\ School of Mathematical Sciences, Queen Mary, University of London, Mile \\ End Road, London E1 4NS, UK
}

\begin{abstract}
To explain the currently observed accelerated expansion of the universe, a large number of different theoretical models are presently being discussed. In one way or another, all of these contain 'new physics', though at different levels. The big question is how to select out of infinitely many possible models the right one. We here discuss a possibility that has so far been somewhat neglected, namely that the new physics underlying dark energy arises out of a gravitationally active amendment of the electroweak and strong sector of the standard model. This amendment basically consists of a rapidly fluctuating gravitationally active dynamics of vacuum fluctuations with a cutoff of the order of the neutrino mass scale. We consider a concrete model for this based on second-quantized self-interacting scalar fields, which evolve in a chaotic way. It is shown that expectations with respect to the chaotic dynamics yield statements on the observed numerical values of the electroweak coupling constants with amazing precision, thus providing evidence for the physical relevance of this model.
\end{abstract}




\section{Introduction}

To understand the fact that the universe is currently in a phase of accelerated expansion 1, 2, 3, 4, an enormous amount of theoretical and experimental work is currently being performed. The favoured explanation for the acceleration is the existence of dark energy, though other possibilities might exist as well. An amazing number of models has been developed in the mean time, and basically every week one finds in the preprint archives some new idea concerning the nature of dark energy. The most popular models are currently quintessence models of various kinds [5], phantom fields [6], Born-Infeld quantum condensates [7], the Chaplygin gas [8], fields with nonstandard kinetic terms [9], to name just a few. All of these approaches contain 'new physics' in one way or another, though at different levels. However, it should also be clear that the number of possible dark energy models that are based on new physics is infinite, and in that sense there is much more to study than the above, currently most popular, models.

When trying to select the most suitable theoretical model for dark energy out of many possibilities, the most straightforward idea would be to compare the various predictions of different theoretical models with the experimental observations (supernovae, cosmic microwave background, large scale structure, etc.) to single out the most relevant model. However, there is a huge degeneracy in the sense that completely different theoretical models cannot be distinguished with the currently available observational data, they often make the same or indistinguishable predictions (e.g. for the equation of state as a function of redshift) or no prediction at all. This situation of degeneracy is not expected to improve significantly in the near future, though the precision of the observational data will increase.

We are thus lead to single out good dark energy models by other, more theoretical criteria. Let us here suggest the following checklist applicable to any dark energy model:

1. Does the model explain why the current value of dark energy density is so small (the cosmological constant problem)?

2. Does the model explain why the current value of dark energy density is of the same order as the matter density (the cosmological coincidence problem) ?

3. Does the model have a reasonable quantum field theoretical background, or does it represent a reasonable extension of quantum field theory?

4. Does the model fit into Einstein's gravity, or does it represent a reasonable extension of it?

5. Is the model compatible with observations? 
6. Does the new physics contained in the model explain some further phenomena that are so far unexplained, for example why the fundamental constants of nature (coupling constants, mass ratios, mixing angles) take on the values we observe and not some other values?

7. Does the model give some sense to dark energy (for example, by explaining it as a relic of inflation), rather than letting it look like an unnecessary curiosity of the universe?

8. Besides dealing with dark energy, does the model also explain why there is dark matter?

9. Is the theory aesthetic and accessible to many physicists, or is it just so complicated that hardly anybody understands it?

10. Are there any laboratory experiments that can verify or disprove this theory?

Every theoretical model builder may rank his or her favourite dark energy model on a scale from $0-10$, depending on how much of the above criteria are satisfied. The perfect theory, from which we are still far away, reaches a mark of 10. Current models, perhaps, reach something in the region $1-5$, at best.

As mentioned before, with the assumption that new physics is relevant there is an enormous number of possible models. In the following I will restrict myself to a model introduced in [10, which scores relatively high on points $3,6,7,10$. With some additional assumptions described in [10, it also scores high on points 1,2 and 4 . The basic idea is that there is a rather 'sterile' amendment of the standard model of electroweak and strong interactions which just consists of a scalar dynamics of vacuum fluctuations with a finite cutoff. The expectation of the underlying potentials produces the currently observed dark energy. Amazingly, the above model seems to distinguish the observed values of the electroweak coupling parameters as local minima in the dark energy landscape. It is well known that quintessence fields can produce a very slow time variation of fundamental constants of nature, e.g. of the fine structure constant [11. Here we go a step further and show that not only a possible variation of the fine structure constant but also its currently observed equilibrium value can be understood by a suitable scalar field dynamics underlying dark energy.

\section{Amending the standard model by gravita- tionally active vacuum fluctuations}

Let us consider the standard model of electroweak and strong interactions. It is a second-quantized field theory and it allows for vacuum fluctuations. The vacuum 
energy density associated with a particle of mass $m$ and spin $j$ is given by

$$
\rho_{v a c}=\frac{1}{2}(-1)^{2 j}(2 j+1) \int \frac{d^{3} k}{(2 \pi)^{3}} \sqrt{\mathbf{k}^{2}+m^{2}}
$$

in units where $\hbar=c=1$. Here $\mathbf{k}$ represents the momentum and the energy is given by $E=\sqrt{\mathbf{k}^{2}+m^{2}}$. Unfortunately, the above integral is divergent. One has to introduce a suitable upper cutoff. Choosing as an upper cutoff the Planck mass $m_{P l}$, one gets an enormous amount of vacuum energy density of the order $m_{P l}^{4}$, larger than the currently observed dark energy density by a factor of $\sim 10^{120}$. This is the famous cosmological constant problem. To circumvent it, the common view is that the absolute value of the above vacuum energy (e.g. in QED) is not observable, it is 'renormalized away', which in a sense means that one adds an infinite constant to get rid of the vacuum energy. This works as long as one does not consider gravity.

However, ultimately we have to unify the standard model with gravity. Also note that almost all particles in the standard model do have mass, so they know what gravity is. It looks a bit like a 'dirty trick' to say that gravity is decoupled from the standard model if the particles have mass. So let us for the moment assume that the vacuum energy in the standard model is cancelled by some kind of symmetry, for example some kind of supersymmetry. This still doesn't explain why we do observe some tiny positive amount of vacuum energy density in the universe, corresponding to the currently measured dark energy density, which is more of the order $m_{\nu}^{4}$ rather than $m_{P l}^{4}$, where $m_{\nu}$ is a typical neutrino mass scale. Hence let us assume that there is something more to the standard model: In addition to the ordinary standard model fields (whose vacuum energies are cancelled by some symmetry) there could be other fields that just show up in a rather sterile way in form of vacuum fluctuations, with a rather small cutoff scale of the order of the neutrino mass. The vacuum energy of these fields is not cancelled by symmetry, there is a symmetry breaking towards positive vacuum energy, at least at the current stage of the universe.

Of course, the above assumption represents new physics, but any decent dark energy model seems to require new physics, in one way or another. The advantages of the above idea are straightforward:

- Since we associate dark energy with a broken symmetry in some sector of this extended standard model, it is not too surprising that the relevant scale of the dark energy density is of the order of some typical particle mass to the power 4 in this model, in this case a neutrino.

- There is increasing experimental evidence [12] for the existence of sterile neutrinos in addition to the known three ordinary neutrino flavours, so apparently there is something more to the standard model than we know. Sterile 
neutrinos may have something to do with the above gravitationally active sector of the standard model.

- Since our dark energy model deals with vacuum fluctuations that are part of the electroweak sector, there is a chance to measure the effects of these fluctuations in laboratory experiments on the earth, such as in Josephson junction experiments, which do probe the spectrum of vacuum fluctuations near the neutrino mass scale due to a nonlinear mixing effect in the junction [13.

In the following, we want to consider a concrete model for vacuum fluctuations with a small cutoff, as introduced in [10]. For quantum field theories with a cutoff, a particular quantization scheme is very convenient to choose, namely the stochastic quantization scheme introduced by Parisi and $\mathrm{Wu}$ [14. This scheme is based on a stochastic differential equation, which naturally embeds various kinds of cutoffs and is by far simpler to deal with than the canonical quantization procedure. For that reason, our gravitationally active amendment of the standard model will be formulated in terms of stochastic quantization. Amazingly, the model will turn out to distinguish the numerical values of the electroweak coupling constants as corresponding to local minima in the dark energy landscape. This can be seen as an indication that one is on the the right track with these kinds of models.

\section{Chaotic model of vacuum fluctuations}

Let us consider a homogeneous self-interacting scalar field $\varphi$ with potential $V(\varphi)$ that forms the basis for our gravitationally active amendment of the standard model. Our amendment should have rather 'sterile' properties, so it is in good approximation sufficient to look at the scalar field equations of this sector on its own, rather than coupling them to the ordinary standard model field equations. We also need the amended sector to consist mainly of vacuum fluctuations with a suitable cutoff, rather than containing stable observable particles, with the possible exception of sterile neutrinos and/or dark matter. In fact, the only connection to the ordinary standard model is that the virtual particles underlying the vacuum fluctuations could potentially interact with the same electroweak and strong coupling constants as in the ordinary standard model.

We quantize the scalar field underlying the steril sector using the Parisi-Wu approach of stochastic quantization. The 2 nd quantized equation of motion is

$$
\frac{\partial}{\partial s} \varphi=\ddot{\varphi}+3 H \dot{\varphi}+V^{\prime}(\varphi)+L(s, t)
$$


where $H$ is the Hubble parameter, $t$ is physical time, $s$ is fictitious time (just a formal coordinate to do quantization) and $L(s, t)$ is Gaussian white noise, $\delta$ correlated both in $s$ and $t$. The fictitious time $s$ is just introduced as a formal tool for stochastic quantization, it has dimensions $\mathrm{GeV}^{-2}$. Quantum mechanical expectations can be calculated as expectations of the above stochastic process for $s \rightarrow \infty$. The simplest way to introduce a cutoff is by making $t$ and $s$ discrete (as in any numerical simulation). Hence we write

$$
\begin{aligned}
& s=n \tau \\
& t=i \delta,
\end{aligned}
$$

where $n$ and $i$ are integers and $\tau$ is a fictitious time lattice constant, $\delta$ is a physical time lattice constant. Note that the uncertainty relation $\Delta E \Delta t=O(\hbar)$ always implies an effective lattice constant $\Delta t$ for a given finite energy $\Delta E$. We also introduce a dimensionless field variable $\Phi_{n}^{i}$ depending on $i$ and $n$ by writing $\varphi_{n}^{i}=$ $\Phi_{n}^{i} p_{\max }$, where $p_{\max }$ is some (so far) arbitrary energy scale. The discretized scalar field dynamics (2) can be written as the following discrete dynamical system [15. 16, 17.

$$
\Phi_{n+1}^{i}=(1-\alpha) T\left(\Phi_{n}^{i}\right)+\frac{3}{2} H \delta \alpha\left(\Phi_{n}^{i}-\Phi_{n}^{i-1}\right)+\frac{\alpha}{2}\left(\Phi_{n}^{i+1}+\Phi_{n}^{i-1}\right)+\tau \cdot \text { noise },
$$

where the local map $T$ is given by

$$
T(\Phi)=\Phi+\frac{\tau}{p_{\max }(1-\alpha)} V^{\prime}\left(p_{\max } \Phi\right)
$$

and $\alpha$ is defined by

$$
\alpha:=\frac{2 \tau}{\delta^{2}} .
$$

For old universes, one can neglect the term proportional to $H$, obtaining

$$
\Phi_{n+1}^{i}=(1-\alpha) T\left(\Phi_{n}^{i}\right)+\frac{\alpha}{2}\left(\Phi_{n}^{i+1}+\Phi_{n}^{i-1}\right)+\tau \cdot \text { noise. }
$$

We now want to construct a field $\varphi_{n}^{i}$ that is different from ordinary fields: Rather than evolving smoothly it should exhibit strongly fluctuating behaviour, so that we may be able to interpret it in terms of vacuum fluctuations. As a distinguished example of a $\varphi^{4}$-theory generating such behaviour, let us consider the map

$$
\Phi_{n+1}=T_{-3}\left(\Phi_{n}\right)=-4 \Phi_{n}^{3}+3 \Phi_{n}
$$

on the interval $\Phi \in[-1,1] . T_{-3}$ is the negative third-order Tchebyscheff map, a standard example of a map exhibiting strongly chaotic behaviour. It is conjugated 
to a Bernoulli shift, and is distinguished as generating the strongest possible chaotic behaviour possible for a smooth low-dimensional deterministic dynamical system [18. The corresponding potential is given by

$$
V_{-3}(\varphi)=\frac{1-\alpha}{\tau}\left\{\varphi^{2}-\frac{1}{p_{\max }^{2}} \varphi^{4}\right\}+\text { const }
$$

or, in terms of the dimensionless field $\Phi$,

$$
V_{-3}(\varphi)=\frac{1-\alpha}{\tau} p_{\max }^{2}\left(\Phi^{2}-\Phi^{4}\right)+\text { const }
$$

The important point is that starting from this potential we obtain by second quantization a field $\varphi$ that rapidly fluctuates in fictitious time on some finite interval, choosing initially $\varphi_{0} \in\left[-p_{\max }, p_{\max }\right]$. Since these chaotic fluctuations are bounded, there is a natural cutoff.

The idea is now that the expectation of the potential of this and similar chaotic fields (plus possibly kinetic terms) underlie the measured dark energy density in the universe. Expectations $\langle\cdots\rangle$ can be easily numerically determined by iterating the dynamics (8) for random initial conditions. One has

$$
\left\langle V_{-3}(\varphi)\right\rangle=\frac{1-\alpha}{\tau} p_{\max }^{2}\left(\left\langle\Phi^{2}\right\rangle-\left\langle\Phi^{4}\right\rangle\right)+\text { const },
$$

which for $\alpha=0$ can be analytically evaluated [10] to give

$$
\left\langle V_{-3}(\varphi)\right\rangle=\frac{1}{8} \frac{p_{\max }^{2}}{\tau}+\text { const. }
$$

Alternatively, we may consider the positive Tchebyscheff map $T_{3}(\Phi)=4 \Phi^{3}-$ $3 \Phi$. It is easy to show that this generates vacuum energy of opposite sign. Symmetry considerations between $T_{-3}$ and $T_{3}$ suggest to take the additive constant const as

$$
\text { const }=+\frac{1-\alpha}{\tau} p_{\max }^{2} \frac{1}{2}\left\langle\Phi^{2}\right\rangle .
$$

In this case one obtains the fully symmetric equation

$$
\left\langle V_{ \pm 3}(\varphi)\right\rangle= \pm \frac{1-\alpha}{\tau} p_{\max }^{2}\left\{-\frac{3}{2}\left\langle\Phi^{2}\right\rangle+\left\langle\Phi^{4}\right\rangle\right\}
$$

which for $\alpha \rightarrow 0$ reduces to

$$
\left\langle V_{ \pm 3}(\varphi)\right\rangle= \pm \frac{p_{\max }^{2}}{\tau}\left(-\frac{3}{8}\right) .
$$


To reproduce the currently measured dark energy, we only need to fix the ratio of the parameters $\tau$ and $p_{\max }$ as

$$
\frac{3}{8} \frac{p_{\max }^{2}}{\tau}=\rho_{\Lambda} \sim m_{\nu}^{4}
$$

This is the simplest model of steril vacuum fluctuations one can think of, a 2nd quantized field theory underlying the cosmological constant $\Lambda$. It is easy to show [10] that for $\alpha=0$ the equation of state of this field is $w=-1$. For small $\alpha$, it is close to $w=-1$. More complicated models, with $w \neq-1$, as well as symmetry breaking breaking between $T_{+3}$ and $T_{-3}$ can be worked out in detail [10. These can produce tracking behaviour of dark energy during the evolution of the universe, and mimic some of the properties of quintessence fields.

\section{Electroweak couplings as local minima in the dark energy landscape}

Let us now give a heuristic argument why the coupling constant $\alpha$ in the above chaotic field equations could have the physical meaning of a gauge coupling. Consider two charges of opposite sign, say, a virtual electron-positron pair, which exists for a short time interval due to a vacuum fluctuation. If the charges are at distance $r$, the Coulomb potential between them is given by

$$
V_{C}(r)=\alpha \frac{1}{r}
$$

(in units where $\hbar=c=1$ ), where $\alpha$ is the fine structure constant. Now for any vacuum fluctuation the inverse distance $1 / r$ is certainly a fluctuating random variable. Motivated by our interpretation of vacuum fluctuations of the field $\varphi_{n}^{i}$ we may choose

$$
\varphi_{n}^{i}-\varphi_{n}^{i-1}=\frac{1}{r}
$$

which has the right dimension and is allowing for both attracting and repelling forces. The above choice basically means that the field difference $\varphi_{n}^{i}-\varphi_{n}^{i-1}$ determines the inverse interaction distance to neighbours in this chaotically evolving discrete model of vacuum fluctuations. Eq. (19) just represents the uncertainty relation $\Delta p \Delta r=O(\hbar)$, interpreting $\varphi_{n}^{i}-\varphi_{n}^{i-1}$ as a momentum uncertainty. Combining eq. (18) and (19), the fluctuating Coulomb potential can thus be written as

$$
V_{C}\left(\varphi_{n}^{i-1}, \varphi_{n}^{i}\right)=\alpha p_{\max }\left(\Phi_{n}^{i}-\Phi_{n}^{i-1}\right)
$$


Summing the two contributions of the pair $(i, i-1)$ and $(i, i+1)$ we just get the linear interaction terms of the nearest neighbours in the discrete dynamics (8) (for more details on this model, see [16], chapter 5). The remarkable thing is that in this interpretation $\alpha$ has now the physical meaning of a gauge coupling. Of course, a similar consideration applies to all kinds of coupling constants (electroweak and strong) in the standard model, not only the fine structure constant. Our central hypothesis is thus that the chaotic fields, though being different from the ordinary standard model fields, interact with the same coupling constants as in the ordinary standard model.

There may be various degrees of freedom of the chaotic fields underlying dark energy. For example, from a dynamical systems point of view, it makes sense to generalize the chaotic field dynamics (86) to

$$
\Phi_{n+1}^{i}=(1-\alpha) T\left(\Phi_{n}^{i}\right)+\sigma \frac{\alpha}{2}\left(T^{b}\left(\Phi_{n}^{i-1}\right)+T^{b}\left(\Phi_{n}^{i+1}\right)\right)
$$

(the noise term can usually be neglected for chaotic maps). The case $\sigma=+1$ is called 'diffusive coupling', the case $\sigma=-1$ 'anti-diffusive coupling'. Chaotic fields with $b=1$ are called to be of 'type A' $\left(T^{1}(\Phi)=: T(\Phi)\right)$, chaotic fields with $b=0$ to be of 'type B' $\left(T^{0}(\Phi)=: \Phi\right)$. There are two different types of vacuum energies for the chaotic fields, namely the self energy $V(\alpha):=\frac{p_{\max }^{2}}{\tau}\left(\frac{3}{2}\left\langle\Phi^{2}\right\rangle-\left\langle\Phi^{4}\right\rangle\right)$ and the interaction energy $W(\alpha):=\frac{p_{\max }^{2}}{2 \tau}\left\langle\Phi_{n}^{i} \Phi_{n}^{i+1}\right\rangle$ (see [17] for more details).

Fig. 1 shows that the self energy $V(\alpha)$ of the chaotic fields indeed distinguishes electroweak coupling constants known from the standard model. Everybody can easily reproduce this plot, by simply iterating the dynamics (8) for random initial conditions $\Phi_{0}^{i} \in[-1,1]$ for a long time on a large lattice and averaging the variable $1.5\left(\Phi_{n}^{i}\right)^{2}-\left(\Phi_{n}^{i}\right)^{4}$. We observe that $V(\alpha)$ has local minima at

$$
\begin{aligned}
& a_{1}=0.000246(2) \\
& a_{2}=0.00102(1) \\
& a_{3}=0.00220(1)
\end{aligned}
$$

( $a_{1}$ and $a_{3}$ are actually small local minima on top of the hill).

On the other hand, in the standard model of electroweak interactions the weak coupling constant is given by

$$
\alpha_{\text {weak }}=\alpha_{e l} \frac{\left(T_{3}-Q \sin ^{2} \theta_{W}\right)^{2}}{\sin ^{2} \theta_{W} \cos ^{2} \theta_{W}}
$$

Here $Q$ is the electric charge of the particle $(Q=-1$ for electrons, $Q=2 / 3$ for $u$-like quarks, $Q=-1 / 3$ for $d$-like quarks), and $T_{3}$ is the third component of the isospin $\left(T_{3}=0\right.$ for right-handed particles, $T_{3}=-\frac{1}{2}$ for $e_{L}$ and $d_{L}, T_{3}=+\frac{1}{2}$ for 


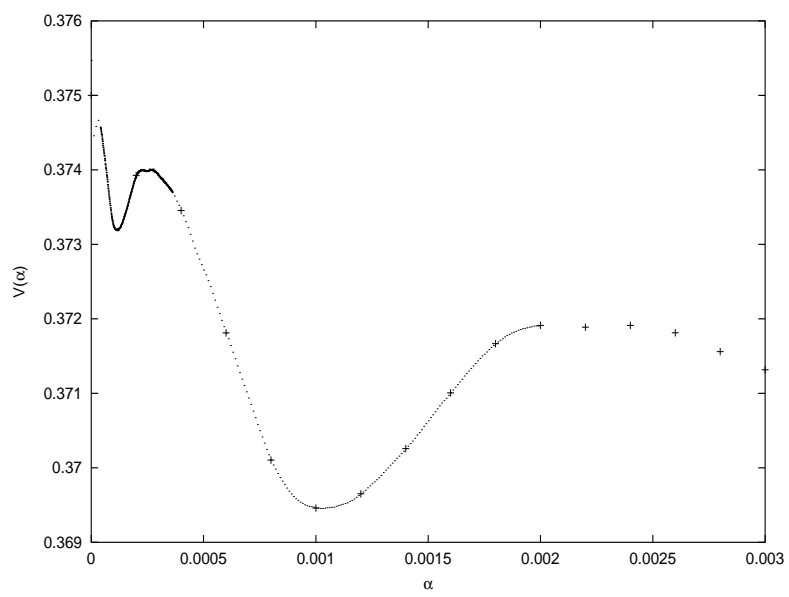

Figure 1: Self energy $V(\alpha)$ of the type-A chaotic field in the low-coupling region. There are local minima at couplings $a_{i}$ that coincide with the weak coupling constants of right-handed fermions in the standard model.

$\nu_{L}$ and $\left.u_{L}\right)$. Consider right-handed fermions $f_{R}$. With $\sin ^{2} \theta_{W}=\bar{s}_{l}^{2}=0.2318$ (as experimentally measured) and the running electric coupling $\alpha_{e l}(E)$ taken at energy scale $E=3 m_{f}$ we obtain from eq. (25) the numerical values

$$
\begin{aligned}
& \alpha_{\text {weak }}^{d_{R}}\left(3 m_{d}\right)=0.000246 \\
& \alpha_{\text {weak }}^{c_{R}}\left(3 m_{c}\right)=0.001013 \\
& \alpha_{\text {weak }}^{e_{R}}\left(3 m_{e}\right)=0.00220 .
\end{aligned}
$$

There is an amazing numerical coincidence between the local minima $a_{1}, a_{2}, a_{3}$ of $V(\alpha)$ and the experimentally measured weak coupling constants of $f_{R}=u_{R}, c_{R}, e_{R}$, respectively. The factor 3 of the energy scale can be related to the index of the Tchebyscheff polynomial [16].

Now regard the fine structure constant $\alpha_{e l}$ and the Weinberg angle $\sin ^{2} \theta_{W}$ as a priori free parameters. Suppose these parameters would change to slightly different values. Then immediately this would produce larger vacuum energy $V(\alpha)$ in our sterile amendment of the standard model, since we get out of the local minima. The system is expected to be driven back to the local minima, and the fundamental parameters are fixed and stabilized in this way.

Further coincidences of this type have been observed for various other observables associated with the chaotic fields, allowing for a fixing of further fundamental constants such as mass ratios and strong couplings at bosonic mass scales. See [16, 17] for details. All these numerically observed coincidences are not explainable as a random coincidence. Rather, they suggest to interpret the coupling 
constant $\alpha$ of our second-quantized chaotic fields $\varphi$ as a running gauge coupling. The chaotic fields are most naturally associated with an additional, sterile sector of the standard model, which just consists of vacuum fluctuations of a scalar field with a cutoff. This sector generates dark energy, and its sense is to fix and stabilize the fundamental constants of nature.

\section{References}

[1] A.G. Riess et al., Astron. J. 116, 1009 (1998), S. Perlmutter et al., Astrophys. J. 517, 565 (1999), J.L. Tonry et al., astro-ph/0305008

[2] N.W. Halverson et al., Astrophys. J. 568, 38 (2002), C.B. Netterfield et al., Astrophys. J. 571, 604 (2002),

[3] D.N. Spergel et al., Astrophys. J. Suppl. 148, 175 (2003) (astro-ph/0302209), C.L. Bennett et al., Astrophys. J. Suppl. 148, 1 (2003) (astro-ph/0302207)

[4] S. Caroll, astro-ph/0004075, S. Weinberg, astro-ph/0005265, T. Padmanabhan, Phys. Rep. 380, 235 (2003) (hep-th/0212290)

[5] P.J.E. Peebles and B. Ratra, Astrophys. J. 325, L17 (1988), C. Wetterich, Nucl. Phys. B312, 668 (1988), M.S. Turner and M. White, Phys. Rev. D56, 4439 (1997), J.A. Frieman and I. Waga, Phys. Rev. D57, 4642 (1998), R.R. Caldwell, R. Dave, and P.J. Steinhardt, Phys. Rev. Lett. 80, 1582 (1998), P.J.E. Peebles and A. Vilenkin, Phys. Rev. D59, 063505 (1999)

[6] R.R. Caldwell, Phys. Lett. B545, 23 (2002), S.M. Caroll, M. Hoffmann, and M. Trodden, astro-ph/0301273, J. Hao and X Li, hep-th/0306033

[7] A.A. Tseytlin, hep-th/9908105, E. Elizalde, J.E. Lidsey, S. Nojiri, and S.D. Odintsov, hep-th/0307177

[8] A.Y. Kamenshchik, U. Moschella, V. Pasquier, Phys. Lett. B511, 265 (2001), M.C. Bento, O. Bertolami, A.A. Sen, Phys. Rev. D66, 043507 (2002)

[9] S. Mukohyama and L. Randall, Phys. Rev. Lett. 92, 211302 (2004) (hep-th/0306108), T. Padmanabhan, T. Roy Choudhury, Phys. Rev. D66, 081301 (hep-th/0205055)

[10] C. Beck, Phys. Rev. D69, 123515 (2004) (astro-ph/0310479)

[11] C. Wetterich, Nucl. Phys. D302, 645 (1988), H.B. Sandvik, J.D. Barrow and J. Magueijo, Phys. Rev. Lett. 88, 031302 (2002) (astro-ph/0107512), C. Wetterich, Phys. Lett. B561, 10 (2003) (hep-ph/0301261), M. Doran, astro-ph/0411606, N.J. Nunes and J.E. Lidsey, Phys. Rev. D69, 123511 (2004) (astro-ph/0310882) 
[12] See, e.g., neutrino reviews at pdg.lbl.gov

[13] C. Beck and M.C. Mackey, Phys. Lett. B605, 295 (2005) (astro-ph/0406505)

[14] G. Parisi, Y. Wu, Sci Sin. 24, 483 (1981), P.H. Damgaard, H. Hüffel (eds.), Stochastic Quantization, World Scientific, Singapore (1988)

[15] K. Kaneko, Progr. Theor. Phys. 72, 480 (1984), R. Kapral, Phys. Rev. A31, 3868 (1985), C. Beck, Phys. Lett. A248, 386 (1998)

[16] C. Beck, Spatio-temporal Chaos and Vacuum Fluctuations of Quantized Fields, World Scientific, Singapore (2002) (50-page summary at hep-th/0207081)

[17] C. Beck, Physica D171, 72 (2002) (hep-th/0105152)

[18] C. Beck and F. Schlögl, Thermodynamics of Chaotic Systems, Cambridge University Press, Cambridge (1993), C. Beck, Nonlinearity 4, 1131 (1991), A. Hilgers, C. Beck, Physica D156, 1 (2001) 